\title{
Analysis of the Possibilities of Applying Mobile Robotic Platforms Using Machine Vision in Industry
}

\author{
Tamara Oleshko ${ }^{1}$, Iryna Heiets ${ }^{2 *}$, Dmytro Kvashuk ${ }^{1}$ \\ 1 Department of Economic Cybernetics, Faculty of Economics and Business Administration, National Aviation University, \\ 03058 Kyiv, 1 Liubomyra Huzara ave., Ukraine \\ 2 School of Engineering, RMIT University, 3000 Melbourne, 124 La Trobe St., Australia \\ * Corresponding author, e-mail: iryna.heiets@rmit.edu.au
}

Received: 15 August 2021, Accepted: 01 October 2020, Published online: 15 November 2021

\begin{abstract}
The article considers the possibilities of automated use of robotic equipment in order to form an infrastructure for moving goods at enterprises. Areas of application of algorithmic programming languages of object-oriented type in robotics are investigated. The algorithm of operation of a transport vehicle, the movement, which is based on the recognition of the line of motion, describing the route of movement, is presented. The analysis of the peculiarities of the implementation of such problems with the use of OpenCv software library was carried out. The structure of the vehicle is proposed, in particular: its driving mechanisms, control scheme, engines and wheelbase. Further development was made to the algorithms for the management of crawler lorries and the ways of their program realization in various spheres of entrepreneurial activity, where there is a need for the transfer of cargoes in the ordinary areas (construction sites, forest lands, open warehouses, airports, etc.). Based on the proposals for creating a cargo robot that can be moved according to a given route, the model of control system of conveyor systems, which solve the issues of automation of technological processes in the part of the addition of conveyor systems, is presented. The analysis of literary sources describing the necessity of creating mobile conveyor systems in production, which enables to quickly re-equip production processes to unforeseen needs, was carried out.
\end{abstract}

Keywords

robotized systems, image recognition, conveyor systems, freight transmissions, programming languages, OpenCv library

\section{Introduction}

The purpose of introducing any automated process control system of technological processes (APCS) is to increase the added value of the final product by reducing and optimizing production costs. The result of such a purpose depends on the means used, their cost and fault-tolerance during operation.

Quite often, market conditions are changing rapidly, new products arise, new services are being created, new components are developed, substitutes for existing raw materials are developed. And sometimes companies are forced to reorient in general to the manufacturing of other products. This requires re-equipment not only of production, but also of transport and warehouse system at the enterprise. In turn, the manufacturer has a fairly high cost for the restructuring of pipelines in particular, and the system for moving loads in general. All this is due to the cost of individual design, individual ordering of components, testing, etc.
Reducing the total cost of both technical maintenance of the control system and the movement of goods within the production site is achieved by increasing the speed and precision of shipment and movement of goods in the process of using the warehouse management system. To this end, in modern conditions, robot-technical solutions of different types are used. The most inexpensive, reliable and simple are crawler-mounted wheeled platforms with independent drives that allow you to maintain the required speed. The engines of the right and left sides can be electric or hydraulic, and the control process is carried out with the help of a manipulation mechanism. There is an opportunity to quickly and effectively incorporate a robotic platform into the general concept of the company's control system.

The most relevant issue related to the use of robotized transport platforms is their independent management during travel, since the performance of this function by 
the general system of APCSs causes an additional load on the system itself, and questions its versatility. The control system should issue commands to the lower-level elements, but under no circumstances should control them. In turn, the requirements for automated vehicles also include universality, compliance with safety standards, and easyto-use features (International Standard IEC). The lower-level element in the control system should adopt a standardized command from the higher-level elements. To solve the management problems by such means, in the era of Internet of Things (IoT), it is quite common to use machine vision for route identification, interference detection, fire detection, smoke signs, and the like, which in general meets the modern requirements for automated systems and makes them more flexible to changes in terms of use. The level of development of software today allows you to easily accomplish such tasks. For the solution of which a methodology is proposed in this article for the introduction of a robotic vehicle into an automated system of conveyor movements that moves according to a given route increasing the possibilities of conveyor lines of production enterprises. An autonomous control of a robotic vehicle can be accomplished by means of machine recognition of a designated line that describes the path of the vehicle, which is applied to its travel route, thus expanding the capabilities of the conveyor systems.

In this regard, the authors put forward a hypothesis about the possibility of using cheap ways to build mobile robotic platforms using machine vision, which includes not only establishing the possibility of building them, but also evaluating the reliability of such solutions.

Since the transportation of such platforms must be autonomous, and the construction of separate specialized routes, even within their own economy, requires significant financial costs, it was decided to manage such a platform using machine vision.

To test this hypothesis, a model of a transport platform was built, which was controlled based on a simple algorithm for tracking the line drawn along the set route.

For the software implementation of the motion algorithm, an interpreter of the Python programming language was used. To apply machine vision, the OpenCv library was used. The hardware is implemented on the basis of a RaspberryPi single-board computer, with battery, electric motors and equipment for managing them. In order to prove the hypothesis for such a platform, a number of models were applied, in particular: models for checking qualification, reliability and performance.

\section{Literature review}

The use of automated mobile platforms has gained widespread popularity in construction (Asadi et al., 2018), agriculture (Aravind et al., 2017), chemical industry (Russell, 1999), education (Mondada et al., 2009) and many other industries.

Today, such platforms often use machine vision for autonomous movement (Pérez et al., 2016; Secuianu et al., 2017; Jing et al., 2019). However, given the complexity of such systems, their cost and serviceability creates a barrier for small businesses. Thus, in particular, problems in the use of mobile platforms arise in the process of adaptation to new routes, as well as communications between such platforms (Jing et al., 2019), autonomous power and recharging (Grimmett, 2015), training such robots (BenAri and Mondada, 2018). All this and many other problems increase the cost of machine vision technology for mobile robotic platforms.

In such conditions, there are many enthusiasts who are trying to reduce the cost of relevant developments. For example, scientists from Romania are trying to create a simple solution for moving a mobile platform based on machine vision (Secuianu and Lupu, 2018), the cost of components (Raspberry Pi 3, ultrasonic sensors, Li-Po battery) and free software (OpenCV, Python, Unix) makes such an offer quite affordable for budget use. Such developments include the following works, for example, to determine the processes of fruit ripening (Mustaffa and Khairul, 2017), the application of mineral fertilizers to plants (Khan et al., 2018), the detection of weeds (Arakeri et al., 2017), and so on.

The largest area of application of such systems is the agricultural complex, where a significant part of farmers are small family households, which actually need such solutions.

Despite the large number of developments, which are based on the above approaches, they largely require individual solutions, because each farm has its own characteristics, and this creates the need for entrepreneurs to master, at least Python programming languages, knowledge of Raspberry Pi operating system Linux, as well as knowledge of basic electronics.

A review of scientific sources revealed that the relevant developments today are using the above components and software.

In addition to that, largely theoretical and applied aspects are covered in the means of open access to information, in particular, the use of the cheap Raspberry $\mathrm{Pi}$ platform and the OpenCv software library to solve machine vision problems. Thus, according to the analysis 
of search queries "OpenCv Raspberry Pi", in the most popular search engine Google, you can get the dynamics (Fig. 1), which indicates the prevalence of such technology, and, in turn, its availability to small businesses.

Therefore, as the literature research shows, an attempt to prove the feasibility of the hypothesis of creating a lowpriced transport platform for small farms is quite likely.

To test the hypothesis about the feasibility of creating a control system for a low-priced mobile transport platform, the authors developed a prototype of it.

\section{Main part}

The problems of the flexibility of conveyor systems are becoming more and more relevant with the advent of e-commerce, the openness of trade routes and other processes of globalization. From time to time, enterprises receive orders for the production, storage and maintenance of new products, but the production facilities do not meet the new requirements for the transfer. Therefore, manufacturers are forced to adapt to new conditions. Sometimes such re-equipment has a higher cost than the profit generated by the innovations.

Among the wide range of transportation systems, it is possible to distinguish crawler platforms that are driven by two independent electric or combustion motors. They are widely used in various fields, both industrial and non-industrial. As a rule, moving objects, such as mobile machines and work, often operate in non-stationary and uncertain conditions. In order to manage such complexes, depending on the specifics of their use, it is necessary to conduct research and develop theoretical and practical provisions for the purpose of creating control systems, which, by adapting to changing conditions, should complement the automated control system by mobile and robotic

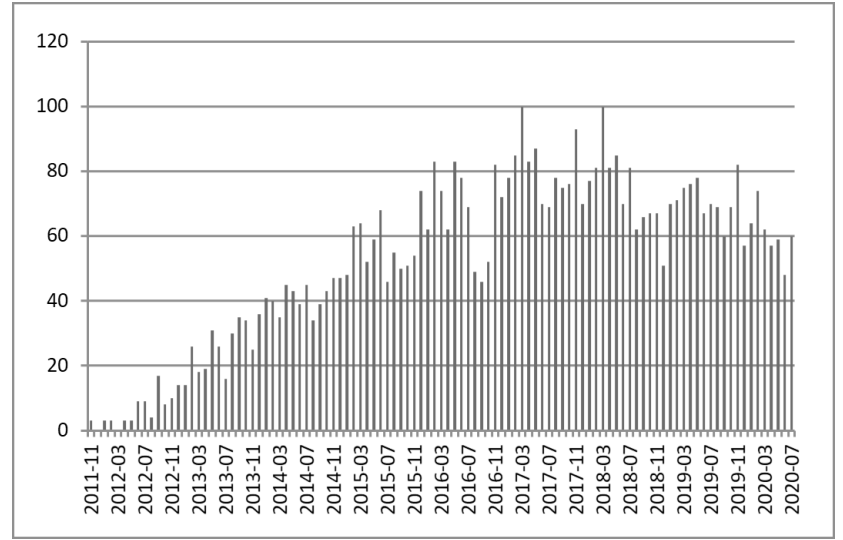

Fig. 1 The number of searches for the keyword "OpenCv Raspberry Pi" in the Google search engine (Compiled according to Google Trends) complexes, which find their application in closed technological cycles (Reimgen, 2014), on separate technological objects (Berger, 2000), in the coal industry (Samorodova et al., 2016), in the chemical industry (Andreeva et al., 2015), and others like that (Glebov and Mohammed, 2015).

A certain complexity is a description of the environment of the working area of the robot, whose parameters are necessary in the programming of its actions, which can be described by Eq. (1).

$$
\begin{aligned}
& v_{0}=r w_{0}\left(1-i_{0}\right) ; v_{i}=r w_{i}\left(1-i_{i}\right) ; \\
& v_{e}=\frac{r}{2}\left[w_{0}\left(1-i_{0}\right)+w_{i}\left(1-i_{i}\right)\right] ; \\
& \varphi=\operatorname{arctg}\left(v_{0}-v_{i}\right) / b ; \\
& v_{e}=\frac{r}{b}\left[w_{i}\left(1-i_{i}\right)+w_{0}\left(1-i_{0}\right)\right] ; \\
& R=\frac{b}{2}\left(w_{0}\left(1-i_{0}\right)+w_{i}\left(1-i_{i}\right)\right) /\left(w_{i}\left(1-i_{i}\right)-w_{0}\left(1-i_{0}\right)\right),
\end{aligned}
$$

where:

- $v_{0}$ - speed of the right caterpillar;

- $v_{i}$ - speed of the left caterpillar;

- $v_{e}$ - translational velocity;

- $r$-driving wheel radius;

- $w_{i}$ and $w_{0}$ - angular velocities of driving wheels;

- $w$ - angular speed of rotation relative to center of mass;

- $\varphi$ - angle of rotation;

- $b$ - the width of the mobile object;

- $R$ - radius of rotation;

- $i_{0}, i_{i}$ - the coefficients of sliding of the right and left caterpillars.

Thus, the cargo of management crawler vehicle (shown in Fig. 2) may be contolled through four control signals:

$$
v_{\text {forward }}=v_{0}+v_{i} \text {; }
$$

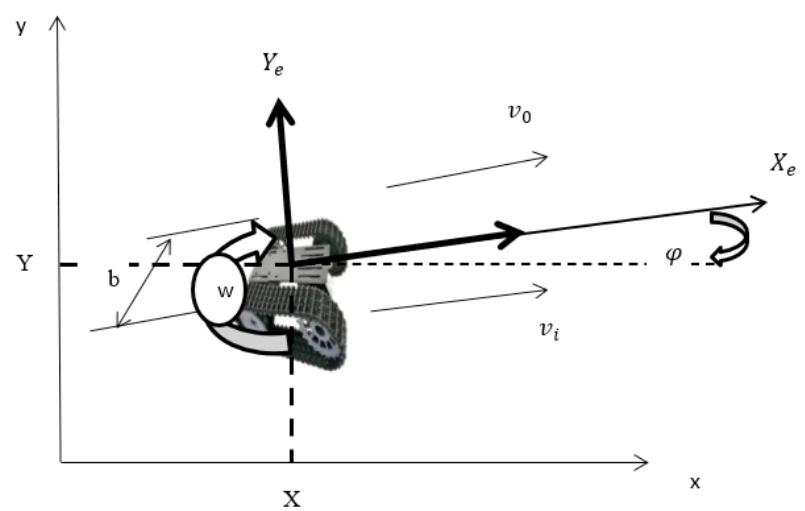

Fig. 2 Scheme of movement of a crawler platform on a plane 


$$
\begin{aligned}
& v_{\text {fback }}=-v_{0}-v_{i} ; \\
& v_{\text {left }}=v_{0}-v_{i} ; \\
& v_{\text {fright }}=-v_{0}+v_{i} ;
\end{aligned}
$$

To set such signals a tracking platform management system based on the received route information is needed. Control signals can be formed on the basis of a computer, on which software for object recognition can be installed.

The following software and hardware composition can be considered as minimum for electric robotic platforms of the crawler type:

- crawler robotic platform with two engines;

- drivers of engines on the four control channels mentioned above;

- power supplies;

- a computer that can install image recognition software and form an output control signal for propulsion of engines;

- a video camera that can transmit video images to a computer;

- safety features;

- alternative channels of robotic platform management in case of failure of existing communication facilities and control systems.

Thus, having these components, the transport platform can be integrated into the general system of control systems. But the necessary conditions for such integration should be autonomy in the process of cargo transportation, reporting to the control system, controllability and safety during operation. In other words, the platform should receive the following commands for stand-alone execution:

- send location relative to the route on request of the control system;

- with a command from the ASTCP, leave from point A to point B and vice versa;

- send a message to the control system about the state of charging of the batteries for their operational replacement;

- if necessary, switch to a separate control mode.

At the request of the ASTC, provide:

1. a statement of emergency,

2. notification of obstacles on the route,

3. notice of transition to a separate management mode.

Send a message to the control system for passing from point $\mathrm{A}$ to point $\mathrm{B}$ and be ready for the next passage of the route. The algorithm of such actions can be represented in a block diagram (Fig. 3).

On the basis of the algorithm of movement, which should guide the transport platform on a given route, it is expedient to apply the technology of recognition of graphic objects, which will enable the recognition of the route of movement.

These graphic objects include a line drawn along the entire route, which must contrast with the movement itself, in order to implement the contour analysis algorithm by the computing device that must be on board the transport platform.

Methods for image recognition can be taken from the OpenCV library, with a license that allows you to do this for free. This library is written in $\mathrm{C} / \mathrm{C}++$ programming languages and is open source. Among the existing analogs, it is most equipped with recognition functions (more than 1000). It was developed in 1998 and has a strong support for the developers community. It can be attributed to the largest library in terms of number of subjects in the processing of images. It has a free BSD license. The license includes only the requirement for the attendant information about this library. Functionality is available in the following languages: Python, Java, C, C ++, CUDA, etc. It is supported by operating systems such as: Linux, Mac, Windows, Android, iOS.

Thus, the expansion of the functionality of the conveyor lines can be represented as follows: the load from the conveyor line enters the robotic platform, then it is delivered from point $A$ to point $B$, where it is passed to the next conveyor line (Fig. 4).

For the automated control of the robotic platform, the Python programming language can be considered the most optimal and easy to master for service personnel. Today it has begun to be used in APCS for various tasks, such as: managing a separate technological process (Merchán et al., 2017), collecting data from industrial sensors (Scholl and Rocha, 2016) and other industries. Therefore, it can be used not only as program elements of the library for image recognition, but also to implement the system as a whole.

The control channels for electric motor drivers and sensor readers can be connected to the GPIOR of the single-chamber mini-computer Raspberry $\mathrm{Pi}$, which is characterized by reliability, possibilities of using a wide range of peripheral devices (camera, monitor, keyboard, etc.), the ability to install free software, including: Linux operating system, Python interpreter, OpenCV library and other software licenses with a free license, and costs about $\$ 50$. The Linux operating system includes direct access to the GPIOR Raspberry Pi port. These options are also 


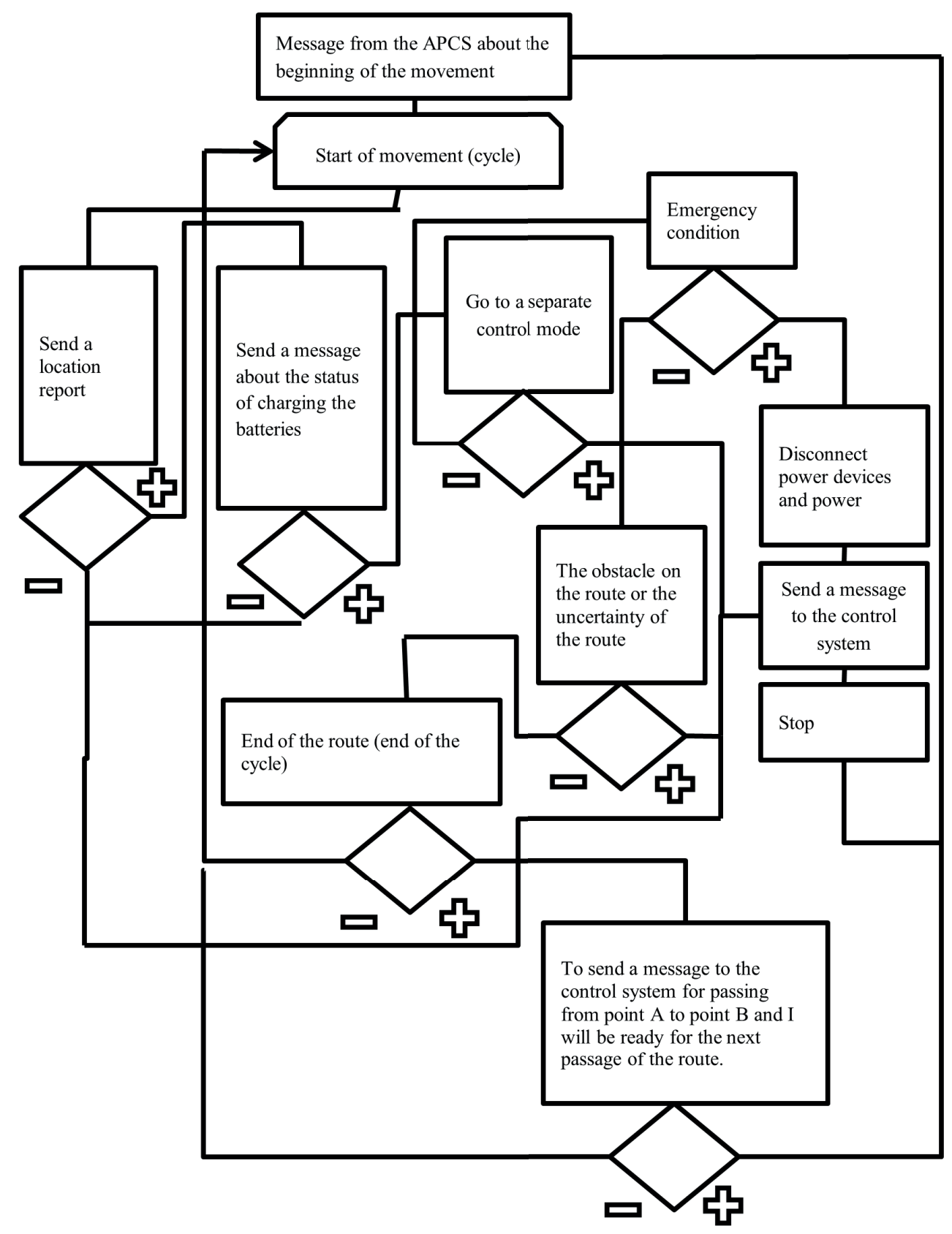

Fig. 3 Robotic platform control algorithm

B

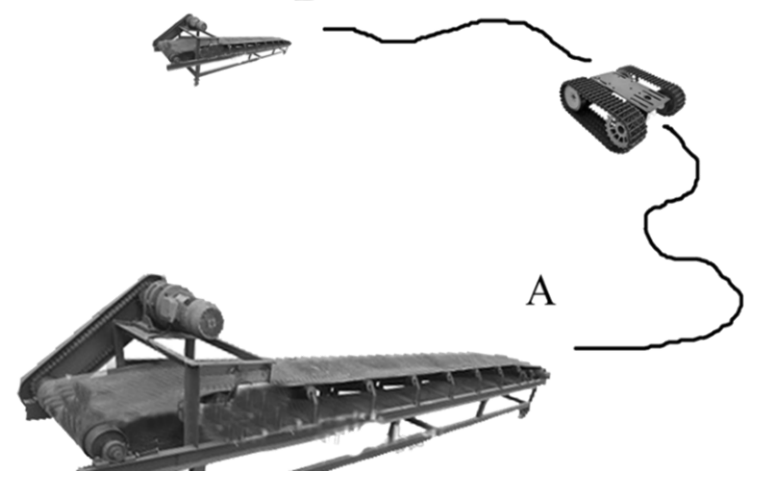

Fig. 4 Expansion of the functionality of conveyor lines using a robotic platform available for the Python interpreter if one uses the RPi. GPIO library or other similar libraries that are supported by this programming language.

So, for determining the route of the transport platform, one can use the OpenCv library, which will enable the recognition of the route followed, the deviation from which will be a signal for proportional control of the control motors.

The code that can be written to implement the platform is simple enough. Given that it is often used in the study of this library, it can be investigated on many examples. The algorithmic problem is reduced to the following points:

1. filtering by color for carrying out the contour analysis of the image; 
2. line separation, filtering of unnecessary parts of the image;

3. detecting the desired line.

Among the many researchers involved in this issue, one can distinguish the works of Shibuya (2017), Forson (2017), Sotnikova (2016), and others. All of them are directed at creating vehicle management systems based on the recognition of individual graphic objects (straight lines, road markings, etc.).

The above tasks, which must be carried out according to the conditions of vehicle movement, have been counted in the program code in the form of various filters, contrast amplifiers, contour smoothing and other settings.

A striking example of these tasks is presented in the Open-Source BeagleBone Black Project, where the developer highlights an easy way to implement such a task in Python (Mabry, 2017).

This code provides the import of a library to work with data arrays that are obtained from rasht images in the form of coordinates of the image contours and the OpenCV library to implement such transformations.

\section{import numpy as $n p$ \\ import cv2}

Video channel settings, using OpenCv library modules, which allow you to select a camera to receive a video image, and set the width and height of the video input. In this case, $160 \times 120$ pixels:

$$
\begin{aligned}
& \text { video_capture }=c v 2 . \text { VideoCapture }(-1) \\
& \text { video_capture.set }(3,160) \\
& \text { video_capture.set }(4,120)
\end{aligned}
$$

The program cycle implements the rest of the code, which captures the current frame of the video, which is cut off the next part of the route line:

$$
\begin{aligned}
& \text { while(True): } \\
& \qquad \begin{aligned}
\text { ret }, \text { frame } & =\text { video_capture.read }() \\
\text { crop_img } & =\text { frame }[60: 120,0: 160]
\end{aligned}
\end{aligned}
$$

In order to highlight the route itself, grayscreens are added and noises are filtered out by the Gauss method, to create a contrast to the image:

$$
\begin{aligned}
& \text { gray }=\text { cv2.cvtColor(crop_img, } \\
& \text { cv2.COLOR_BGR2GRAY) } \\
& \text { blur = cv2.GaussianBlur(gray, (5,5),0) }
\end{aligned}
$$

Converts an image to black and white to form an array of contour points by setting the border between shades. In this case, with 255 colors, all those that are smaller than the 60th order are white and the rest are black:

$$
\text { ret, thresh }=c v 2 \text {.threshold }
$$

(blur,60,255,cv2.THRESH_BINARY_INV)
Using the find contours function, which takes a copy of an image that already has a clear contrast, the outlines of the route line are highlighted.

$$
\begin{aligned}
& \text { contours, hierarchy }= \\
& \text { cv2.findContours(thresh.copy(), 1, } \\
& \text { cv2.CHAIN_APPROX_NONE) }
\end{aligned}
$$

The condition for finding contours is checked and, if found, the largest of them are determined by the max function, and then the coordinate of this contour is located (in this example, $c x$ and $c y)$.

$$
\begin{aligned}
& \text { if len(contours) }>0: \\
& c=\max (\text { contours, key }=c v 2 . \text { contourArea) } \\
& M=c v 2 . \text { moments }(c) \\
& c x=\operatorname{int}\left(M\left[{ }^{\prime} m 10^{\prime}\right] / M\left[' m 00^{\prime}\right]\right) \\
& c y=\operatorname{int}\left(M\left[' m 01^{\prime}\right] / M\left[' m 00^{\prime}\right]\right)
\end{aligned}
$$

Contour lines are applied to the original image. cv2.line (crop_img, $(c x, 0),(c x, 720),(255,0,0), 1)$ cv2.line (crop_img, (0, cy), (1280, cy), $(255,0,0), 1)$ cv2.drawContours (crop_img, контуры, $-1,(0,255,0)$, 1)

Now the robot defines the position of the line on the basis of which it directs the controlling effect on its engines. In the case of a line definition, a signal is generated.

$$
\begin{aligned}
& \text { if } c x>=120 \text { : } \\
& \text { print ('Turn Left!') } \\
& \text { if } c x<120 \text { and } c x>50 \text { : } \\
& \text { print ('On Track!') } \\
& \text { if } c x<=50: \\
& \text { print ('Turn Right') } \\
& \text { else: } \\
& \text { print ('don't see the line') }
\end{aligned}
$$

The resulting video can be displayed in the graphic window of the operating system, as well as realizing the exit from the cycle when one presses any button on the keyboard.

$$
\begin{aligned}
& \text { cv2.imshow('frame', crop_img) } \\
& \text { if cv2.waitKey(1) \& } 0 x F F==\operatorname{ord}\left({ }^{\prime} q\right. \text { '): break }
\end{aligned}
$$

Thus, the use of mobile robotic platforms at production facilities based on the management of machine vision technologies has a wide range of opportunities for the implementation of tasks for the improvement of the control system, optimization of production, expansion of capacities of conveyor lines and reduction of expenses for unplanned re-equipment of the enterprise.

\section{Conclusion}

Object-oriented programming languages, with the development of computing power are increasingly entangled in the control system. This applies not only to the general management system. Thus, in the proposed example, the 
programming language Python describes the wide possibilities for controlling the functions of the machine-vision library and the robotic control of the platform, as well as the expediency of its use in APCS.

The proposed directions of optimization of the control system using mobile robotic platforms managed on the basis of line identification, which can be applied to almost any surface, can greatly simplify the internal logistics of enterprises, allow integration of such platforms into the overall enterprise management system, as well as expand the functionality of conveyor lines.

In the example presented, a general concept of the formation of an internal automated transport infrastructure, which can be further developed, depending on the needs and characteristics of the enterprise, is proposed.

Testing of the proposed prototype of a robotic mobile platform confirms the hypothesis that low-priced transport platforms can be used for the small farms. This will allow further progress of the developments proposed in the article in order to improve the working conditions

\section{References}

Andreeva, M. M., Shagapov, I. L., Soloviev, N. V., Nuretdinov, I. I. (2015) "Современные Scada-Системы в Химико-Технологической Промышленности" (Modern SCADA-systems in the chemical-technological industry), Bulletin of Kazan Technological University, 18(2), pp. 312-315. [online] Available at: https://cyberleninka.ru/article/n/sovremennye-scada-sistemy-v-himiko-tehnologicheskoy-promyshlennosti/viewer [Accessed: 15 July 2020] (in Russian)

Arakeri, M. P., Vijaya Kumar, B. P., Barsaiya, S., Sairam, H. V. " Computer vision based robotic weed control system for precision agriculture", In: 2017 International Conference on Advances in Computing, Communications and Informatics (ICACCI), Udupi, India, 2017, pp. 1201-1205.

https://doi.org/10.1109/ICACCI.2017.8126005

Aravind, K. R., Raja, P., Pérez-Ruiz, M. (2017) "Task-based agricultural mobile robots in arable farming: A review", Spanish Journal of Agricultural Research, 15(1), Article number: e02R01. https://doi.org/10.5424/sjar/2017151-9573

Asadi, K., Ramshankar, H., Pullagurla, H., Bhandare, A., Shanbhag, S., Mehta, P., ..., Wu, T. (2018) "Building an Integrated Mobile Robotic System for Real-Time Applications in Construction", In: 35 th International Symposium on Automation and Robotics in Construction (ISARC), Berlin, Germany, pp. 453-461 https://doi.org/10.22260/ISARC2018/0063

Ben-Ari, M., Mondada, F. (2018) "Elements of robotics", Springer Nature, Cham, Switzerland. https://doi.org/10.1007/978-3-319-62533-1

Berger, H. (2000) "Automating with STEP 7 in LAD and FBD", Publicis Publishing, Erlangen, Germany. of small businesses in agriculture. As part of the experiment, $80-90 \%$ stability of the prototype was obtained for a number of models in the evaluation of the reliability of technological equipment, which indicates its effectiveness. Such solutions can significantly simplify the internal logistics of enterprises, integrate such platforms into the overall enterprise management system, and expand the functionality of conveyor lines.

Thus, the proposed mobile robotic platforms, which are managed based on line identification, can be applied to almost any surface, thereby significantly simplifying the internal logistics of the small farms, allowing the integration of such platforms into the overall management system of such enterprises, as well as expanding the functionality of conveyor lines.

\section{Acknowledgement}

The project presented in this article is supported by National Aviation University (Ukraine) and RMIT University (Australia).

Forson, E. (2017) "Recognizing Traffic Signs With 98\% Accuracy Using Deep Learning" [online] Available at: https://towardsdatascience.com/recognizing-traffic-signs-with-over-98-accuracy-using-deep-learning-86737aedc2ab [Accessed: 13 July 2020]

Glebov, N. A., Mohammed, A. N. (2015) "Control of autonomous mobile tracked vehicles and robots in the non-formalized medium", University News, North-Caucasian Region, 2015(3), pp. 17-21. https://doi.org/10.17213/0321-2653-2015-3-17-21

Grimmett, R. (2015) "Raspberry Pi Robotics Projects", Packt Publishing Ltd., Birmingham, UK.

Jing, P., Zheng, W., Xu, Q. (2019) "Vision-based Mobile Robot's Environment Outdoor Perception", In: The 3rd International Conference on Computer Science and Application Engineering (CSAE 2019), Sanya, China, Article number: 120. https://doi.org/10.1145/3331453.3361655

Khan, N., Medlock, G., Graves, S., Anwar, S. (2018) "GPS Guided Autonomous Navigation of a Small Agricultural Robot with Automated Fertilizing System", SAE Technical Paper, 2018-01-0031, SAE International, Warrendale, PA, USA. https://doi.org/10.4271/2018-01-0031

Mabry, A. (2017) "Building a Line Following BeagleBone Robot with openCV", [computer program] Available at: http://einsteiniumstudios.com/beaglebone-opencv-line-following-robot.html [Accessed: 11 July 2020]

Merchán, D. F., Peralta, J. A., Vazquez-Rodas, A., Minchala, L. I., Astudillo-Salinas, D. (2017) "Open Source SCADA System for Advanced Monitoring of Industrial Processes", In: 2017 International Conference on Information Systems and Computer Science (INCISCOS), Quito, Ecuador, pp. 160-165. https://doi.org/10.1109/INCISCOS.2017.9 
Mondada, F., Bonani, M., Raemy, X., Pugh, J., Cianci, C., Klaptocz, A., ..., Martinoli, A. (2009) "The e-puck, a Robot Designed for Education in Engineering", In: 9th Conference on Autonomous Robot Systems and Competitions, Castelo Branco, Portugal, pp. 59-65. [online] Available at: https://infoscience.epfl.ch/record/135236 [Accessed: 11 July 2020]

Mustaffa, I. B., Khairul, S. F. B. M. (2017) "Identification of fruit size and maturity through fruit images using OpenCV-Python and Rasberry Pi", In: 2017 International Conference on Robotics, Automation and Sciences (ICORAS), Melaka, Malaysia, pp. 1-3. https://doi.org/10.1109/ICORAS.2017.8308068

Pérez, L., Rodríguez, Í., Rodríguez, N., Usamentiaga, R., García, D. F. (2016) "Robot Guidance Using Machine Vision Techniques in Industrial Environments: A Comparative Review", Sensors, 16(3), Article number: 335. https://doi.org/10.3390/s16030335

Reimgen, Y. (2014) "Automated process control systems", Scada system, Part 1, Mining information and analytical bulletin (scientific and technical journal), 8, pp. 386-393.

Russell, R. A. (1999) "Odour Detection by Mobile Robots", World Scientific Publishing, Singapore, Singapore. https://doi.org/10.1142/4042

Samorodova, L., Lyubimov, O., Yakunina, Y. (2016) "Application of SCADA Systems in the Coal Mining Industry", In: 8th RussianChinese Symposium "Coal in the 21st Century: Mining, Processing, Safety", Kemerovo, Russia, pp. 50-54.

https://doi.org/10.2991/coal-16.2016.10
Scholl, M. V., Rocha, C. R. (2016) "Embedded SCADA for Small Applications", IFAC-PapersOnLine, 49(21), pp. 246-253. https://doi.org/10.1016/j.ifacol.2016.10.559

Shibuya, N. (2017) "Finding Lane Lines on the Road", [online] Available at: https://towardsdatascience.com/finding-lane-lines-on-theroad-30cf016a1165 [Accessed: 11 July 2020]

Secuianu, F. D., Lupu, C. (2018) "Implementation of a home appliance mobile platform based on computer vision: self-charging and mapping", In: The 22nd International Conference on System Theory, Control and Computing (ICSTCC), Sinaia, Romania, pp. 464-468. https://doi.org/10.1109/ICSTCC.2018.8540685

Secuianu, F., Mihai, C., Vulpe, A., Lupu, C. (2017) "Implementation of an autonomous mobile platform based on computer vision", In: The 18th International Carpathian Control Conference (ICCC), Sinaia, Romania, pp. 246-251. https://doi.org/10.1109/CarpathianCC.2017.7970405

Sotnikova, M. V. (2016) "Алгоритм автоматического удержания колесного робота на визуально заданной линии" (Algorithm for visual path following by wheeled fully actuated mobile robot), Bulletin of St. Petersburg University, Series 10, Applied Mathematics, Computer science, Management processes, 2016(1), pp. 99-108. [online] Available at: http://www.mathnet.ru/php/ archive.phtm1 wshow $=$ paper $\&$ jrnid $=$ vspui\&paperid=281\&op tion_lang=rus [Accessed: 08 July 2020] (in Russian) 